\title{
Slit and integral-field optical spectroscopy of the enigmatic quasar HE 0450-2958*
}

\author{
G. Letawe ${ }^{1}$, P. Magain ${ }^{1}$, and F. Courbin ${ }^{2}$ \\ 1 Institut d'Astrophysique et Géophysique, Université de Liège, Allée du 6 Août, 17 Bâtiment B5C, 4000 Liège, Belgium \\ e-mail: gletawe@ulg.ac.be \\ ${ }^{2}$ Laboratoire d'Astrophysique, École Polytechnique Fédérale de Lausanne (EPFL), Observatoire, 1290 Sauverny, Switzerland
}

Received 24 September 2007 / Accepted 3 December 2007

\begin{abstract}
Context. Interest in the quasar HE 0450-2958 arose following the publication of the non-detection of its expected massive host, leading to various interpretations.

Aims. This article investigates the gaseous and stellar contents of the system through additional VLT/FORS slit spectra and integral field spectroscopy from VLT/VIMOS.

Methods. We apply our MCS deconvolution algorithm on slit spectra for the separation of the QSO and diffuse components, and develop a new method to remove the point sources in Integral Field Spectra, allowing extraction of velocity maps, narrow-line images, spatially resolved spectra or ionization diagrams of the surroundings of HE 0450-2958.

Results. The whole system is embedded in gas, mostly ionized by the QSO radiation field and shocks associated with radio jets. The observed gas and star dynamics are unrelated, revealing a strongly perturbed system. Despite longer spectroscopic observations, the host galaxy remains undetected.
\end{abstract}

Key words. galaxies: active - quasars: individual: HE 0450-2958

\section{Introduction}

In the framework of a systematic spectroscopic study of 20 QSO host galaxies at low redshift (Letawe et al. 2007), we discovered a particularly interesting object with no detected host galaxy: HE 0450-2958. While all the other QSOs in the sample show a host galaxy even in the short acquisition exposures taken with the ESO Very Large Telescope (VLT), that of HE 0450-2958 remains undetected, both on our deep VLT optical spectra and on high resolution ACS images taken with the Hubble Space Telescope (HST). From these observations Magain et al. (2005, hereafter M05) set a deep upper limit, implying that, given the luminosity of the QSO, the host galaxy is underluminous by at least a factor of six.

HE 0450-2958, at $z=0.285$, lies in a rather complex environment, as shown in Fig. 1. Aside from a foreground star which happens to be close to the line of sight, about 1.7" to the North-West of the QSO, two other objects are at the same redshift as the QSO: 1- a strongly distorted companion galaxy, located at $1.5^{\prime \prime}(6.5 \mathrm{kpc})$ to the South-East of the QSO; 2- a compact blob whose spectrum shows prominent emission lines, typical for highly ionized gas, but with no trace of continuum light. This gas is most probably ionized by the QSO itself, as suggested by the emission lines flux ratios (M05).

Since the publication by Magain et al. (M05), several papers have proposed alternative explanations to the fact that no stellar light is detected around HE 0450-2958. In particular, the QSO could have been ejected during a 3-body

\footnotetext{
* Based on observations made with VLT/UT1 (program 76.B0693(B)), and with the VLT/UT3 (program 72.B-0268(B)) at ESO-Paranal observatory in Chile, in addition with NASA/ESA Hubble Space Telescope observations from Cycle 13, proposal \#10238.
}

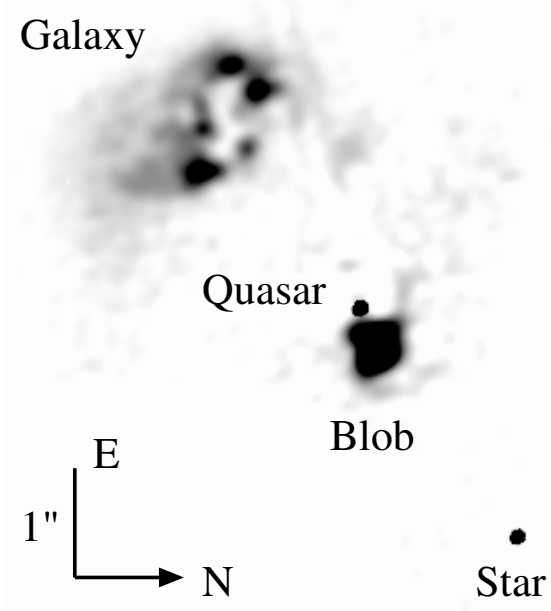

Fig. 1. HST/ACS image of HE 0450-2958, obtained with the High Resolution Channel of the instrument, through the F606W filter. The image has been spatially deconvoled using the MCS algorithm (adapted from M05).

interaction, where a binary black hole kicked the QSO off during a merger event (Hoffman \& Loeb 2006). An alternative ejection mechanism would be recoil due to gravitational wave emission (Haehnelt et al. 2006). Although this scenario is supported by the recent discovery of a genuine triple QSO (Djorgovski et al. 2007), further simulations tend to weaken these hypotheses (Merritt et al. 2006). In their analysis Merritt et al. note that HE 0450-2958 emission lines are narrower than 
for the majority of Type I QSOs. They suggest HE 0450-2958 to be a high luminosity analog to the so-called narrow-line Seyfert 1 galaxies (NLS1s), where a lower-mass supermassive black hole (SMBH) accretes matter at a high rate, possibly above the Eddington limit. This is indeed supported by X-ray analyses (Zhou et al. 2007). If the empirical relation between the mass of the SMBH and the mass of the host galaxy bulge holds (e.g. Marconi \& Hunt 2003), one would then expect a lower luminosity host galaxy for HE 0450-2958, possibly just below the detection limit of M05. Estimates of the host magnitude upper limits were also performed using a different method by Kim et al. (2007), confirming the value of M05. These authors argue, in agreement with Merritt et al. (2006), that the bulge might be just too faint for detection with the observations made so far.

Other possible explanations for the non-detection of the host are internal extinction by dust and/or the absence of a genuine young stellar population. In both cases, the host galaxy might be at the limit of detection of the M05 ACS images. To date, the only evidence suggesting the presence of a host comes from the radio observations of Feain et al. (2007). These authors point out that the observed fluxes in different radio bands are consistent with star formation-induced emission and, moreover, that they obey the Far Infrared/Radio Continuum correlation found for star forming galaxies.

The present paper focuses on new long slit and integral field spectroscopy carried out at the ESO-VLT, with the aim of characterizing the physical state of the gas surrounding HE 0450-2958 and also to attempt to detect the stellar continuum of the host galaxy, if it exists.

\section{Observations and reductions}

\subsection{Slit (2D) spectroscopy with VLT/FORS2 MXU}

Our new multi-slit observations of HE 0450-2958 were obtained with the ESO-VLT and the FORS2 instrument in the MXU (mask exchange unit) mode. We used the G600RI grism, covering the spectral range from 5145 to $8470 \AA$, i.e., the major stellar and gas emission lines in the redshifted spectrum of HE 0450-2958. The observations were taken between February and April 2006, as 5 sets of 6 different exposures, for a total exposure time of $3.75 \mathrm{~h}$ in dark time. Their spectral resolving power is $R=1000$.

The slitlets of the MXU mask are all 1" wide, but have variable lengths. As in M05 and Letawe et al. (2007) we place slitlets on the quasar and on several surrounding stars of similar magnitude. The spectra of the stars are used as PSF templates to remove the quasar light using the version of the MCS algorithm (Magain et al. 1998) for spatial deconvolution of spectra (Courbin et al. 2000). The orientation of the slits is selected to allow the simultaneous observation of the quasar, the companion galaxy and the foreground star. The remaining slits are used to observe the surrounding galaxies. The seeing during the observations varied from 0.5 to $1.3^{\prime \prime}$.

The basic reductions are performed with IRAF tasks, leading to flatfielded, wavelength calibrated and sky subtracted spectra, with $1.5 \AA /$ pixel in the spectral direction and $0.126^{\prime \prime} /$ pixel in the spatial direction.

In addition to the slit spectra, several images of the field were taken with FORS2 to design the slit masks. Three 30-s exposures were taken for this purpose, through the $B, V$ and $R$ filters. Figure 2 shows the combination of these three exposures, with two different intensity contrasts to enhance both faint and bright

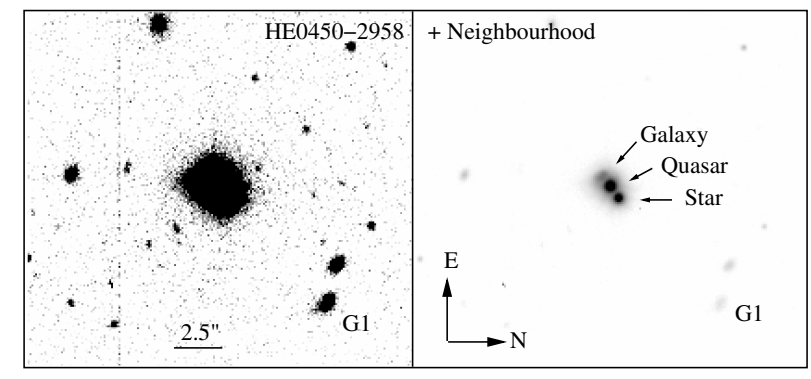

Fig. 2. Immediate surrounding of HE 0450-2958. The image is the combination of the 30-s exposures in $B, V$ and $R$ obtained at the VLT/FORS2 to design the slit masks. Two different intensity scales are shown to display the full dynamical range of the image.

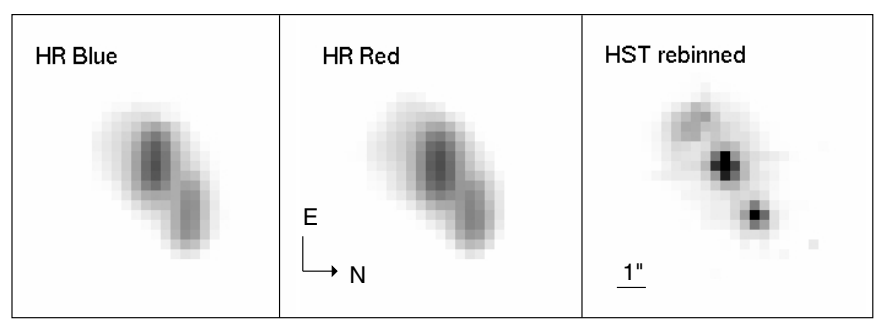

Fig. 3. VIMOS reduced data integrated over the whole spectral range, for the HRblue (left) and HRred grisms (middle). For comparison we show the HST/ACS image on the right panel, rebinned at the same orientation and resolution as the VIMOS data. Intensity scales are logarithmic.

structures. They reveal many faint objects within a few arcseconds of HE 0450-2958. However, as the quasar and most point sources of similar brightness are saturated, no further data processing is carried out on these images.

\subsection{Integral field spectroscopy (3D) with VLT/VIMOS}

The integral field unit (IFU) of the VIMOS instrument was used to obtain 3D spectra of HE 0450-2958. In this configuration, a spectrum is obtained for each position on a $13^{\prime \prime} \times 13^{\prime \prime}$ field of view, every $0.33^{\prime \prime}$. The spectral resolution is $R \sim 2500$.

Two settings (HRblue and HRred) are used to cover the wavelength range $4200 \AA<\lambda<8600 \AA$. Unfortunately, the gap in wavelength between the two grisms (6150 to $6350 \AA$ ) contains the $\mathrm{H} \beta$ emission line, at the redshift $(z=0.285)$ of HE 0450-2958. The spectra provided by the optical fibers fed by the telescope are organized in four quadrants containing $20 \times 20$ fibers each.

The observations consist of $7 \times 40 \mathrm{~min}$ exposures with the blue grism in December 2003, and of $11 \times 19$ min with the red grism, taken between December 2003 and February 2004. The seeing varied from 0.45 to $0.9^{\prime \prime}$ in the blue $\left(0.63^{\prime \prime}\right.$ on average) and from 0.53 to $1.2^{\prime \prime}$ in the red grism ( $0.76^{\prime \prime}$ on average).

At the time of observation, the HRred grism was not available on the fourth quadrant and was replaced by HRorange, covering a bluer wavelength range and with a different response curve. This complicates the analysis of the fourth quadrant. As a consequence, only 7 out of the 11 HRred exposures were useful due to problem in the matching of HRred and HRorange on the edges of the fourth quadrant, located at the NW corner of the field (see Fig. 3). In this figure, we show the VIMOS field of view in the two settings and we compare it with the HST/ACS image degraded to the resolution and pixel size of VIMOS. The VIMOS PSF is highly elongated. Since our reduction procedure 
(see below) corrects for atmospheric refraction, this elongation is probably due to a tracking problem.

The observations are reduced using various pipelines and routines, taking advantage of the specific capabilities of each package. The main steps of the reduction can be summarized as follows:

- the cosmic-ray rejection is carried out on the raw data by applying the filter/cosmic task of MIDAS to the 2D spectra in each of the four quadrants;

- the bias subtraction, flatfielding, wavelength calibration, extraction of the spectra for all fibers, and flux calibration are done using the ESOREX recipes from the VIMOS Pipeline provided by ESO. The reduced data with the HRblue grism have a spectral sampling of $0.54 \AA$ /pixel and the data taken with the HRred grism have $0.58 \AA /$ pixel;

- a basic 2D sky subtraction is carried out with the background IRAF task, by linear interpolation on each spectral element at positions devoid of sources;

- whenever a strong sky emission line is not removed well enough by the IRAF task, a FORTRAN routine is applied to clean the spectrum by linear interpolation between the regions unaffected by these sky emission lines;

- PYTHON routines are then used to re-organize the spectra into $3 \mathrm{D}$ data cubes of $40 \times 40$ spatial resolution elements each containing a 1D spectrum. We use the word "spaxels" in the following, when referring to each of the $40 \times 40$ elements, to avoid confusion with pixels;

- the reduced exposures are then aligned using a PYTHON task, which shifts the spatial location of the quasar for each spectral element to the center of the IFU field. This ensures that atmospheric refraction is corrected in each exposure;

- the aligned exposures are co-added with appropriate weighting, taking into account the different coverage of the sky due to offsets between exposures;

- the resulting combined data cubes are smoothed using a Gaussian kernel of $2.5 \AA$ full width at half maximum $(F W H M)$.

\section{Slit spectroscopy}

Because of the very high contrast between the QSO and its putative host galaxy, particular care must be paid to the decomposition of the data into point-like and extended components, both in the slit and IFU spectra.

The FORS2/MXU spectra of HE 0450-2958 are all deconvolved using the MCS algorithm for slit spectra (Magain et al. 1998; Courbin et al. 2000), allowing a separation of the QSO light from the underlying extended objects. This method has been used successfully on many occasions, either on data of QSO host galaxies (Courbin et al. 2002; Letawe et al. 2004; M05; Letawe et al. 2007) or on data of gravitationally lensed QSOs (e.g., Eigenbrod et al. 2006, 2007).

The goal of the present observations was to go deeper than in M05, to attempt the detection of a stellar continuum in the putative host galaxy and to determine the ionization state of the gas far away from the QSO. Unfortunately, optimal separation of the QSO and host cannot be achieved in this case: the seeing during the observations, worse than originally planned, is of the same order as the slit width, resulting in a loss of information on the wings of the PSF. This effect is enhanced by the combined effects of a slight slit misalignment with respect to the point sources, and of differential atmospheric refraction. The consequence is that the QSO and the PSF stars are clipped in

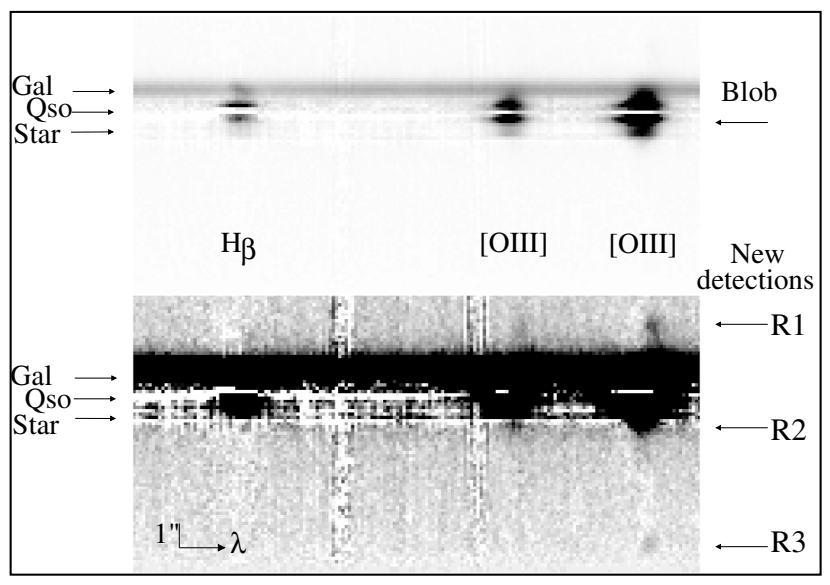

Fig. 4. Combined FORS2 spectrum of HE 0450-2958, in the region where the deconvolution shows acceptable residuals. The point sources (QSO+star) have been removed. The spectra are shown with different intensity scales in the two panels. The 3 new emission line regions are indicated. The source of their ionization is the QSO radiation.

different ways, making it impossible to build reliable PSFs. In addition, the overall quality of the PSF does not allow simulations to be performed (see M05) in order to place a useful limit on the magnitude of the continuum.

We show in Fig. 4 a representative portion of the data, between $\mathrm{H} \beta$ and [OIII], where the deconvolution process is acceptable, i.e., a region where the remaining flux after removal of the point sources is non-negative, and where the residuals of the deconvolution process do not present obvious features of a misadapted PSF. The image is the combination of all 5 of our individual, deconvolved, spectra. Even with bad deconvolution residuals, as shown by the consequent noise at the position of the point sources, and thanks to the deep exposures, three new emission-line regions are detected at large distances from the QSO (see Fig. 4).

The three regions $\mathrm{R} 1, \mathrm{R} 2$, and $\mathrm{R} 3$, are located at 2.6, 1.5 and 5.9 arcsec from the QSO. This corresponds to projected distances of 12,7 and $27 \mathrm{kpc}$ respectively, at the redshift of HE 0450-2958. While regions R1 and R3 had not been detected previously, region R2 was reported in Feain et al. (2007), on the basis on our FORS1/MOS analysis (M05). It is almost superimposed on the foreground star lying to the North-West of the QSO.

Only emission lines are seen in the 3 regions, despite the depth of the observations. In the 3 cases, the [OIII] doublet is detected while the $\mathrm{H} \beta$ line is absent. For region R1, this converts into a lower limit of $[\mathrm{OIII}] / \mathrm{H} \beta>7.7$, and for region $\mathrm{R} 2$, $[\mathrm{OIII}] / \mathrm{H} \beta>28.8$. The third region is so faint that the lower limit derived is irrelevant. Following the ionization diagrams of Veilleux \& Osterbrock (1987), a ratio above 6 can safely be associated with ionization by QSO radiation. Our FORS2 observations therefore indicate that the source of ionization in these regions is the QSO itself and, as a consequence, that ionizing photons can escape the QSO surroundings to ionize gas clouds as far as at least 7 and $12 \mathrm{kpc}$. This gives further support to the idea that, if dust is present in the immediate surroundings of the QSO, its geometry is such that UV radiation can escape freely in several directions: towards us (no detected reddening of the QSO and blob spectra, M05) and towards these emission line regions.

There is still no detection of a host galaxy in these spectra but, as previously mentioned, the quality of the deconvolution process is not optimal. However, a comparison of the flux in the 


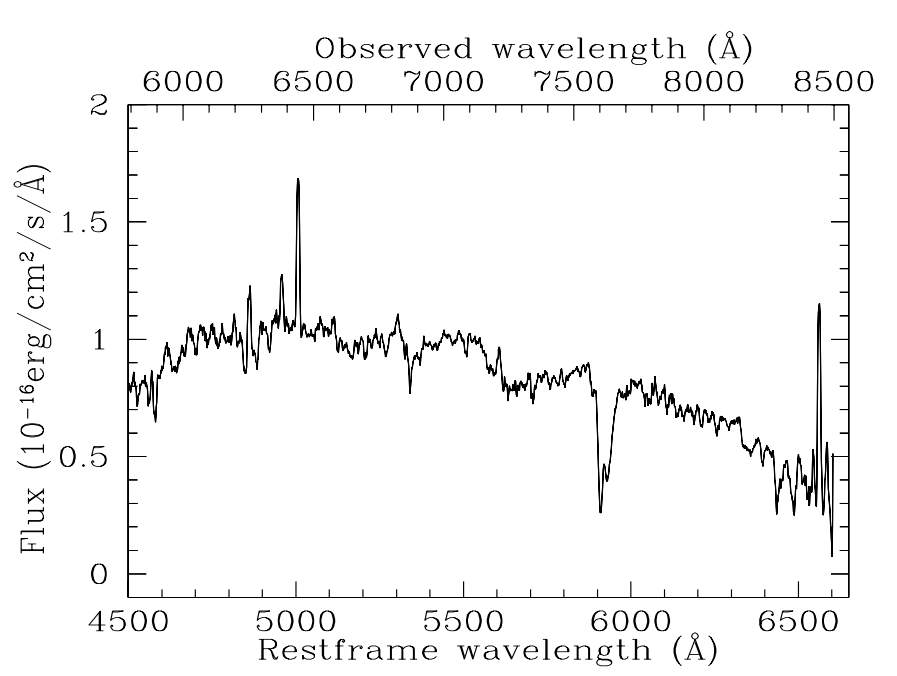

Fig. 5. FORS2 spectrum of the late-type galaxy G1 shown in Fig. 2. The measured redshift is $z=0.2866$.

companion galaxy and the remaining flux under the QSO (i.e. the putative host) reveals that the host should at least be two magnitude fainter than the companion galaxy, in the wavelength range between $[\mathrm{OIII}]$ and $\mathrm{H} \alpha$ (equivalent to the observed filter $R$ ). This would give a magnitude for the host of $M_{V} \sim-21$, compatible with M05 and Kim et al. (2007) estimates. Given that most of the flux detected under the QSO is likely to be due to deconvolution artefacts caused by an inaccurate PSF, the putative host might be much fainter than that upper limit.

Finally, some of the slits used for these observations were placed on galaxies close to the line of sight to HE 0450-2958. One of them is galaxy G1 of Fig. 2, for which we obtain the spectrum shown in Fig. 5. The redshift of this late-type galaxy is close to that of the quasar, $z=0.2866$. The projected linear distance between G1 and the quasar is $44 \mathrm{kpc}$. No other related galaxy was found in these and the previous FORS 1 spectra (Letawe et al. 2007).

\section{Integral field (3D) spectroscopy}

\subsection{Subtraction of the point sources}

Integral field spectroscopy (IFS) allows investigation of the immediate surroundings of the QSO, provided its own spectrum can be subtracted from the data. Because of the lack of isolated stars in the VIMOS field of view, and because of the elongation of the PSF, deconvolution of the data, as done with slit spectra, is not possible. Instead, we use another approach, taking advantage of the 3D information we have at our disposal.

First, we select a reference spectrum at a central position for each point source, i.e., the QSO and the foreground star of Fig. 1. The two spectra are used as templates to be removed from the other spaxels, after adequate weighting. As there is a clear variation of the grism+detector response function accross the array of fibers, the weighting is allowed to vary linearly with wavelength, for each spaxel.

The relative weighting of the QSO and of the stellar templates are considered as satisfactory when there is no trace left, in a given spaxel, of the Balmer broad emission lines from the QSO and of the (broad) absorption lines for the star. Visual inspection of the residuals is carried out for every spaxel contaminated by a point source, allowing us to make sure that the result of the subtraction does not show signs of over- or undersubtraction, at any wavelength. This procedure leaves us with a data cube containing only the companion galaxy and any other extended objects such as regions of ionized gas.

The method described above relies on the important assumption that the QSO template actually contains only light from the QSO, which might not be the case. Our template probably contains a small but non-zero contribution of (narrow) gas emission lines not belonging to the QSO itself. We may therefore systematically oversubtract the emission lines in each spaxel, in particular for the "blob", which lies angularly close to the QSO. Moreover, the subtraction significantly increases the noise in the regions where the QSO or the star dominate the flux, i.e. close to the centers of their PSFs.

The adopted procedure for point source subtraction being based on visual inspection of the removal of spectral point source characteristics, the error bars are difficult to quantify precisely. However, as we aim to subtract the broad components with no condition on narrow ones, the presence of narrow emission lines in the subtracted spectra is reliable, and might just be slightly underestimated by the possible subtraction of part of the blob spectrum along with the quasar template. The continuum slope might also be affected by a wrong weighting of the template spectra, so we did not use these slopes to determine any properties of the emitting regions.

\subsection{Extracted spectra}

The result of the QSO+star subtraction is displayed in Fig. 6. In this figure, we also divide the field of view into different regions where we integrate the signal. Three main areas are defined and then subdivided into smaller areas: the region of the companion galaxy, the more central region of the "blob", and several regions further away from the QSO, which also turn out to contain ionized gas.

\subsubsection{The companion galaxy}

The companion galaxy can be subdivided into three areas which we name Gall to Gal3 (Fig. 6). The corresponding 1D spectra are displayed in Fig. 7. While the spectrum of region Gall is dominated by young stars (important continuum, weak [OIII] lines, broad Hydrogen absorptions), region Gal2 is a mix of star and gas, and region Gal3 is almost exclusively made of ionized gas. As the smearing of light is of consequence in the VIMOS data, we deliberately integrated the signal over large regions, which have about the length-scale of the seeing. The matching between the regions and the HST/ACS broad-band image is shown in Fig. 6. Clearly, regions of highly ionized gas extend beyond the broad-band image of the galaxy: there is basically no light in the HST/ACS image of the Gal3 region, as well as no continuum in its spectrum.

\subsubsection{The blob}

The region of the blob is probably the most difficult to interpret on the VIMOS data, as it is located very close to the reference fiber taken to model our QSO template spectrum. Although the subtraction process may bias the spectrum of the blob (essentially by removing part of it together with the QSO spectrum), the present results support the M05 findings that the blob is made only of gas and shows no trace of a stellar continuum. The 1D spectrum of this region is shown in Fig. 8. 

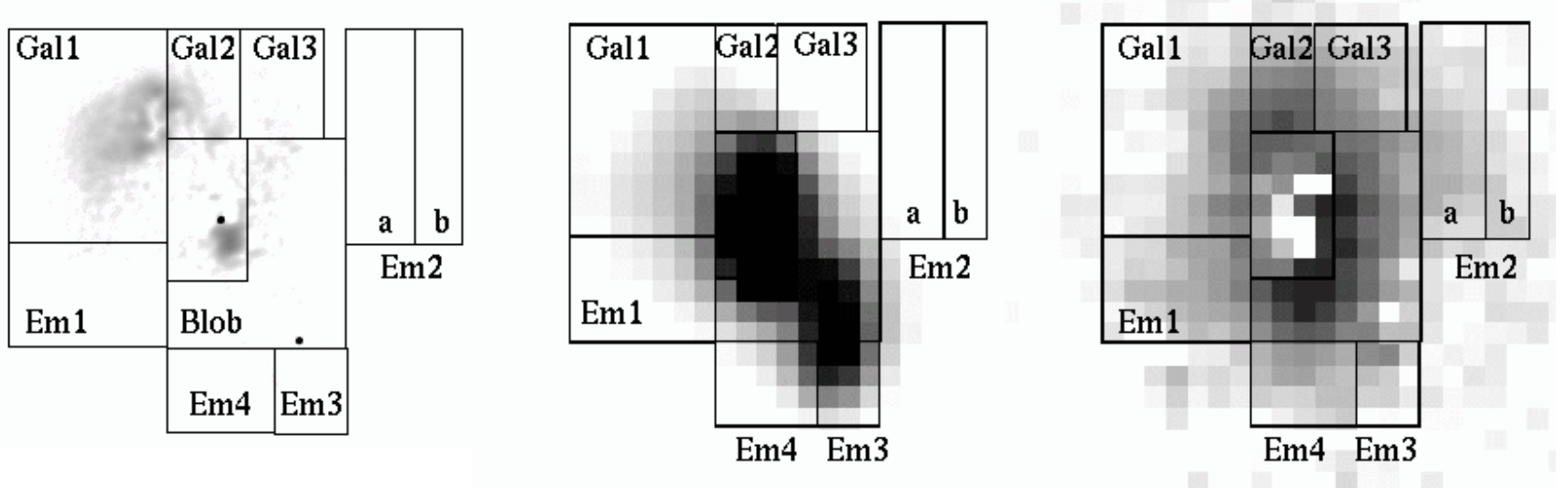

Fig. 6. Spatial regions over which we integrate individual 1D spectra. The regions are shown in overlay on the deconvolved HST/ACS image (left panel), and on the VIMOS image reconstructed from the full datacube by integrating over the full wavelength range (middle panel). The image on the right is the result of the subtraction of the QSO and of nearby star spectra. In this case, the wavelength range around the redshifted $[\mathrm{OII}]$ emission line has been selected. The central 30 spaxels of the QSO, very noisy after subtraction, are not used when integrating the signal over the regions.

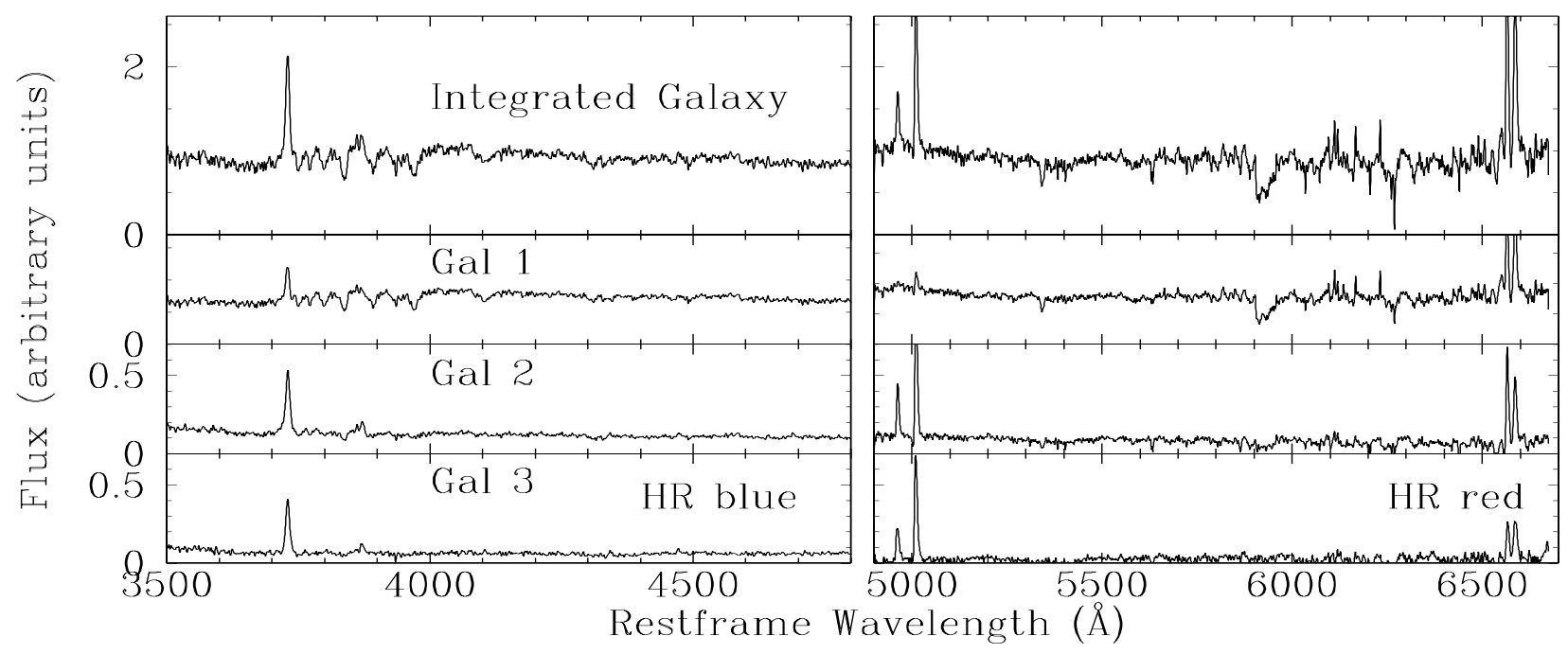

Fig. 7. Spectra of the companion galaxy in the HRblue and HRred VIMOS grisms. For the two settings, the spectrum in the top panel is the sum of the spectra for the 3 regions labeled Gal1 to Gal3 in Fig. 6, and shown in the lower panels.

Note that even if our subtraction procedure dimmed the continuum of the blob, it has also dimmed the emission lines. The fact that the latter are still very bright after subtraction of the QSO spectrum shows that we have not completely removed the signal.

\subsubsection{Emission line clouds}

The four regions labelled Eml to Em4 in Fig. 6 are dominated by emission lines. Their integrated 1D spectra are shown in Fig. 9. All emission lines are real, as no appropriate weighting of the subtracted nuclear spectra can make them disappear, even when trying to oversubtract the templates. At the depth of the observations, only Eml contains both gas and stars, as can be seen from the non-negligible $4000 \AA$ break. This region might be an extension of the companion galaxy. The three other regions are compatible with gas emission only. The weird continuum of Em3 is caused by its proximity to the center of the foreground star, these spaxels being more affected by the PSF variation with position and wavelength. Nothing can be seen at the position of Em2, Em3 and Em4 in the HST/ACS image (Fig. 6, left). Em3 probably corresponds to the faint gaseous emission R2 in the VLT MXU spectra of Fig. 4.

\subsubsection{A 2D diagnostic diagram}

Diagnostic diagrams such as the ones introduced by Veilleux \& Osterbrock (1987) and Baldwin et al. (1981) allow identification of the nature of the source of ionization in a gas cloud. These diagrams involve emission line ratios sensitive to the hardness of the ionizing flux. Our VIMOS data allow us to map some of these ratios accross the field of HE 0450-2958.

We show in Fig. 10 a color-coded 2D diagnostic diagram for the regions in the vicinity of HE 0450-2958. The 3-color scale in the right panel gives the ionization source as a function of color. The three main sources of ionization are labeled in the figure as "AGN" for ionization by strong power-law source (here the QSO), "HII" when the ionization is due to stars, and "shock" for shock-induced ionization.

In order to be accurate, the line ratio must be corrected for absorption by dust. The lack of an $\mathrm{H} \beta$ line in these spectra makes it hard to carry out this correction and hampers the use of the 


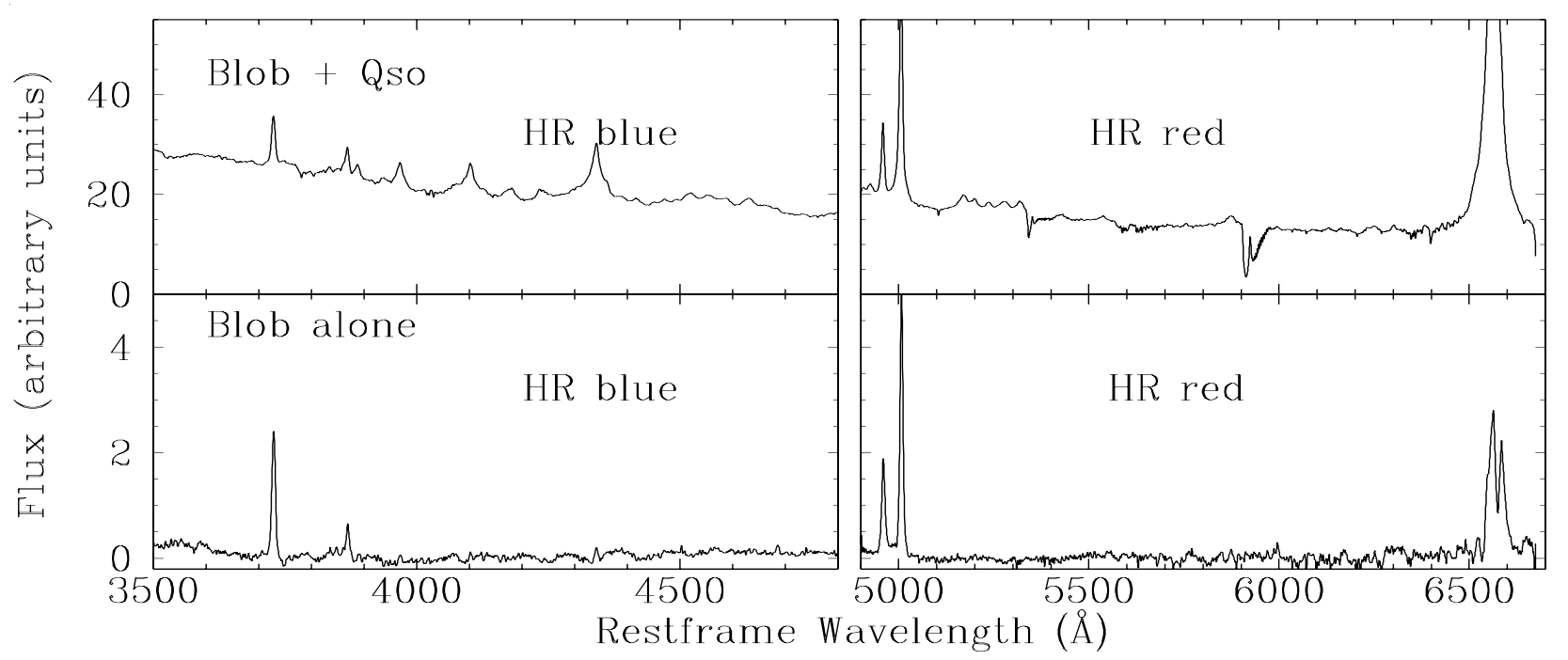

Fig. 8. Spectra corresponding to the "blob" region in the HRblue and HRred grisms. The spectra are shown before and after the QSO is removed, in the upper and lower panels respectively.

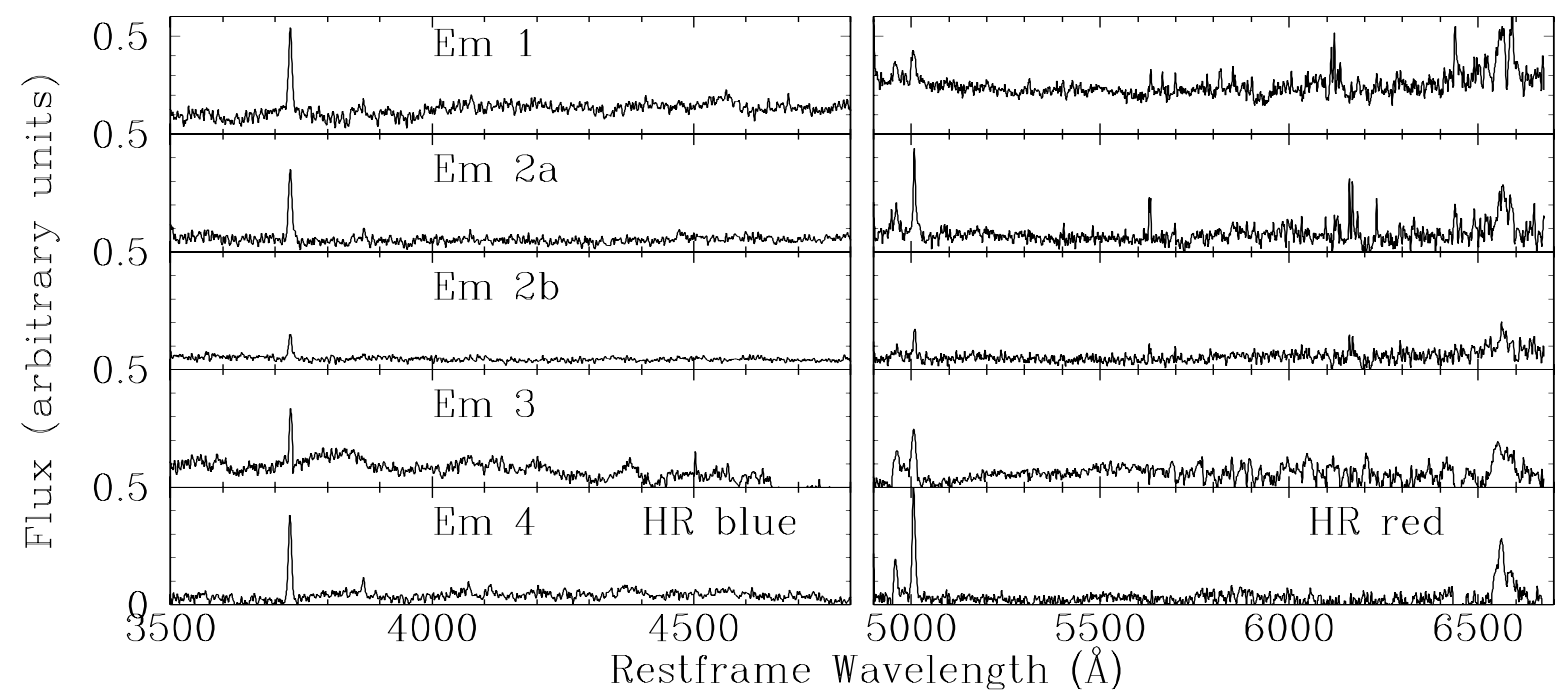

Fig. 9. Spectra corresponding to the regions labelled Eml to Em4 in Fig. 6, all dominated by ionized gas.

line ratio proposed by Veilleux \& Osterbrock (1987). However, our diagnostic diagram is constructed using the $[\mathrm{NII}] / \mathrm{H} \alpha$ and $[\mathrm{OII}] /[\mathrm{OIII}]$ line ratios, as presented in Baldwin et al. (1981). With these ratios, any reddening leaves the $[\mathrm{NII}] / \mathrm{H} \alpha$ unchanged, as the two lines are almost at the same wavelength, but decreases the $[\mathrm{OII}] /[\mathrm{OIII}]$ ratio: $[\mathrm{OII}]$ being at shorter wavelength than [OIII], it is much more affected by dust absorption.

The vast majority of spaxels are found in the AGN-ionized region (blue dominant). They concentrate in the blob, and in the regions Gal2/3 and Em3/4. The Southern part of the system $($ Gall/Eml) is ionized by shocks and stars. Following Balmer decrements evaluated on FORS1 slit spectra (M05), the companion galaxy is the only place where dust reddening is non negligible. However, a correction for this extinction, increasing $[\mathrm{OII}] /[\mathrm{OIII}]$, would not affect the dominance of the shockinduced ionization.

As the ionization source of the blob is clearly the quasar light, the possible oversubtraction of a part of the blob (narrow component) along with the quasar template spectrum (as explained in Sect. 4.1) possibly lowers the hard ionization across the entire system. The dominance of AGN-caused ionization measured in the whole system may thus be even stronger than suggested by Fig. 10.

The only regions where the ionization by star dominates, are the region $E m 2 b$ and the outer edge of region Gall, where the signal is very weak. Even if we find almost no continuum in the weak Em2 region, this might indicate the presence of young stars even outside the companion galaxy. The distribution of the green shock-induced ionization regions in Fig. 10, on each side of the QSO, suggests that the stars were formed during a violent event, probably triggered by the QSO (or together with the QSO) and not in a global, continuous star formation in the companion galaxy.

Finally, note that the spectra of the different Em zones are very faint, and that $\mathrm{H} \alpha$ and $[\mathrm{NII}]$ are not well separated in those regions (see Fig. 9), resulting in large uncertainties on the measured ratios. Still, the measured [NII]/H $\alpha$ would have to be wrong by an order of magnitude to change our main conclusion that the gas is ionized by a hard radiation field, probably that of the QSO. 

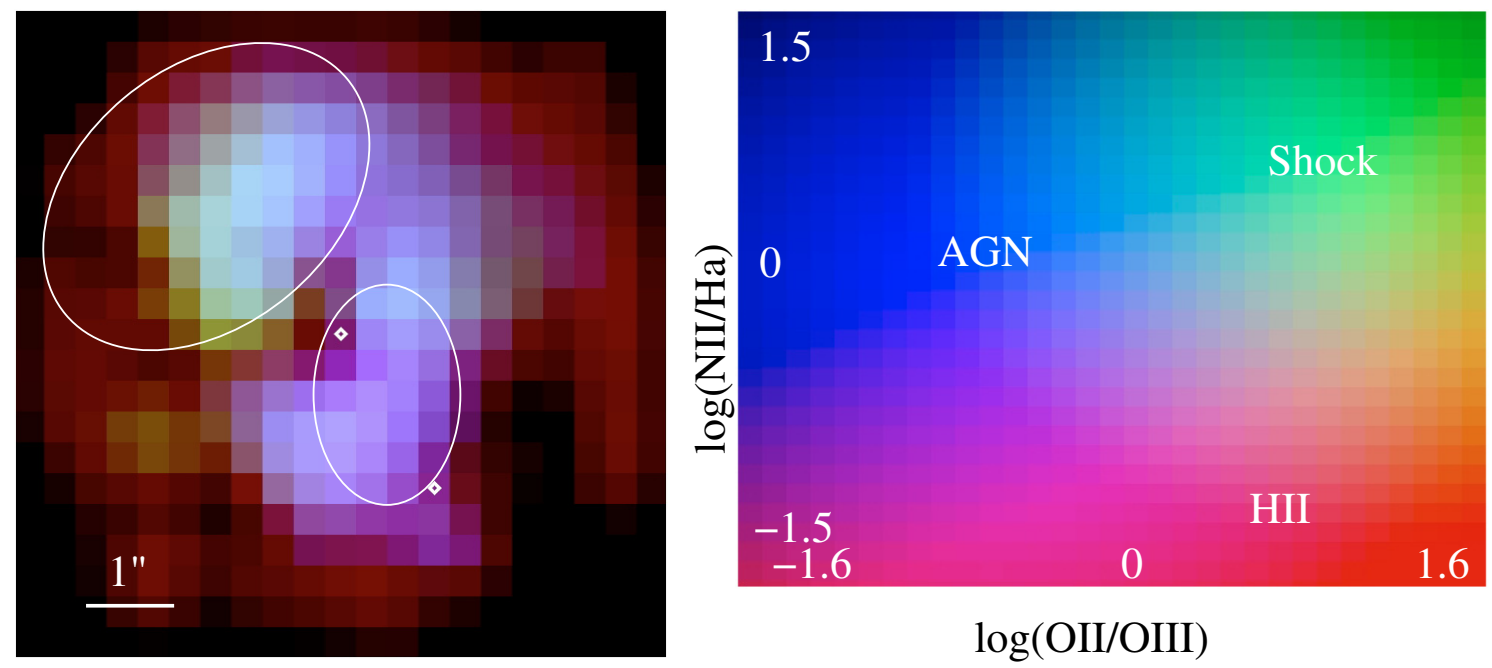

Fig. 10. Color-coded diagnostic diagram constructed from the VIMOS emission line images after subtraction of the QSO and of the nearby star, as in the right panel of Fig. 6. The positions of the QSO and of the nearby star are indicated by white diamonds, while the companion galaxy and the "blob" are indicated by white ellipses. The color code in the image is defined in the right panel. The field of view is $7 \times 7$ ", i.e., a zoom on the central part of the VIMOS field. The image has been slightly smoothed, and weighted at each position by the corresponding emission intensity.

\subsection{Emission line images}

We use our VIMOS data to produce narrow-band images of HE 0450-2958, centered on several emission lines: [OII]3727 $\AA$, $[\mathrm{OIII}] 4959+5007 \AA$, and $\mathrm{H} \alpha 6563 \AA+[\mathrm{NII}] 6583 \AA$. For each line, we subtract an image in the continuum around the emission line, after appropriate normalization. We also show an image of the continuum between $[\mathrm{OII}]$ and $\mathrm{H} \beta$, a wavelength range devoid of any emission lines. We overlay on Fig. 11 the contours of emission lines images on the deconvolved HST/ACS data, where point sources are indicated by dots. We set to zero the noisy pattern near the position of the QSO, because of the large uncertainty due to the removal of its spectrum at this location.

We observe a slight shift between the position of the blob in the HST image and in the VIMOS contour plots. The reason for this is twofold: first, as we take a reference spectrum at the central position for PSF subtraction, we remove part of the underlying extended component (i.e. the blob) together with the quasar light at those positions. Second, the spatial resolution of VIMOS is much lower than that of ACS, so small emission regions in the HST data are spread on much larger areas in the VIMOS data. One spaxel in the VIMOS data is about the size of the blob.

The most striking differences between the spatial distribution in the various spectral bands are given below:

- the continuum light nicely follows the ACS image of the companion galaxy. No continuum is found at the position of the blob. Only small and faint structures are seen in the vicinity of the QSO and are all compatible with the noise;

- the [OII] emission is present in the companion galaxy, the blob, and extends all around HE 0450-2958. This line arises both in star forming regions and in gas clouds ionized by AGN light. Its extended distribution indicates that the system seems globally embedded in gas. Note that the effect is not due to an arbitrary choice of the intensity scales: although the $[\mathrm{OII}]$ envelope is fainter than the [OIII] emission (see, e.g., Fig. 9), it extends much further away from the QSO;

- the [OIII] doublet needs harder ionizing photons than [OII], such as ionization by an AGN. In Fig. 11, [OIII] is essentially present in the blob, in the Northern part of the companion galaxy, as well as close to the position of the foreground star.

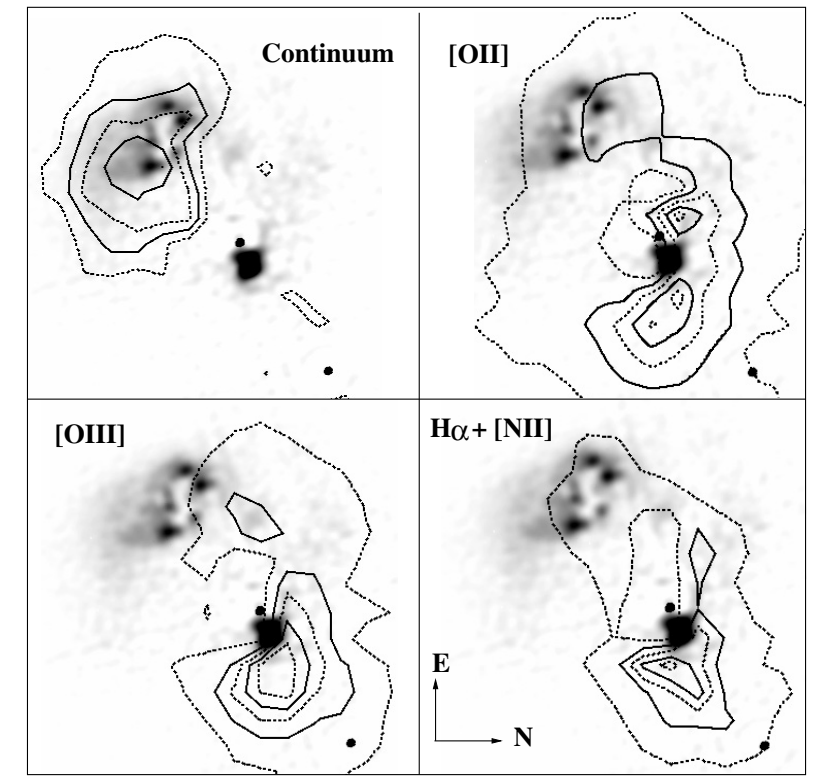

Fig. 11. Contours plot for the continuum VIMOS image (see text) and for different emission lines. The contours are shown in overlay of the HST/ACS image, after subtraction of the QSO and of the nearby star. We alternate solid and dotted contours for clarity of display. The steps between contours are every $20 \%$ of the maximum intensity of each image.

- the $\mathrm{H} \alpha$ distribution is more extended than [OIII] in the companion galaxy, probably because of ongoing star formation. The blob is also visible in this line.

\subsection{Velocity maps}

The velocity maps of Fig. 12 are constructed by fitting a Gaussian profile to the emission lines in each spaxel. The velocities are then given relative to the companion galaxy average velocity, as estimated from the Gall emission lines. The velocity contours obtained in this way are then slightly smoothed. With 


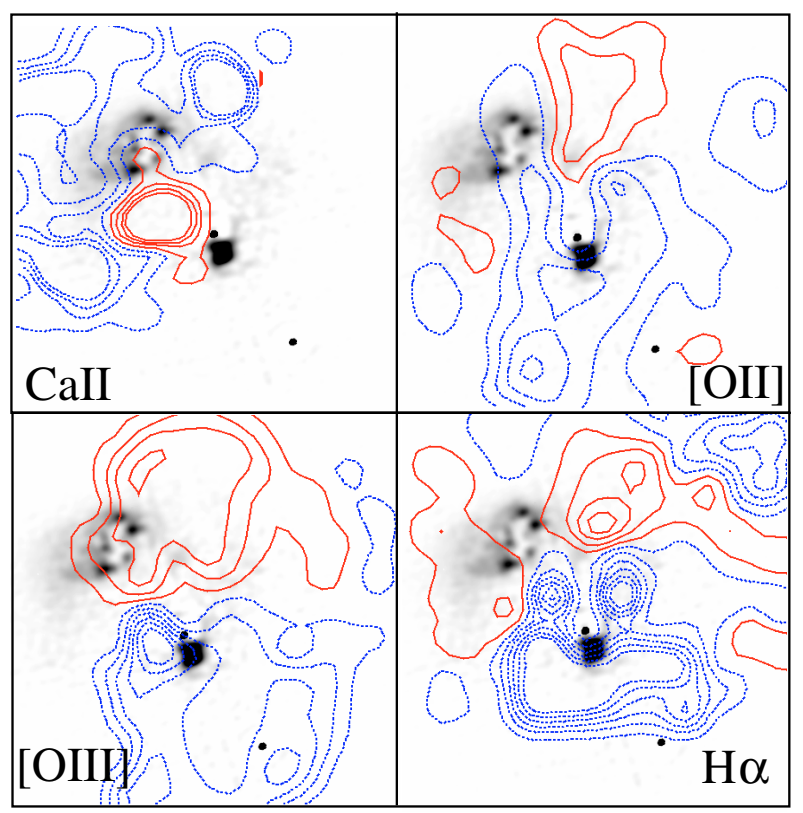

Fig. 12. Velocity maps for the stellar and gas components, overlaid on the HST/ACS deconvolved image. The velocity contours are relative to the companion galaxy systemic velocity. The blue dashed contours are negative, and the red solid contours are positive. Top left: CaII absorption line velocity contours, from -200 to $+200 \mathrm{~km} \mathrm{~s}^{-1}$ with steps of $55 \mathrm{~km} \mathrm{~s}^{-1}$. Top right: [OII] emission line velocities, where the contours are drawn from -190 to $+160 \mathrm{~km} \mathrm{~s}^{-1}$ by steps of $50 \mathrm{~km} \mathrm{~s}^{-1}$. Bottom left: the $[\mathrm{OIII}]$ emission line, with contours from -290 to $+160 \mathrm{~km} \mathrm{~s}^{-1}$ with steps of $50 \mathrm{~km} \mathrm{~s}^{-1}$. Bottom right: $\mathrm{H} \alpha$ emission, from -290 to $+160 \mathrm{~km} \mathrm{~s}^{-1}$ with steps of $60 \mathrm{~km} \mathrm{~s}^{-1}$. See text for interpretations.

the setting of the VIMOS observations, we can map both the gas and stellar velocity fields.

The best lines to trace the gas in our observations are [OII] and [OIII]. The $\mathrm{H} \alpha$ velocity map is noisier because of the high nucleus-to-host ratio in this line. Spaxels in the immediate surrounding of the quasar position are masked. The velocities derived from these lines range from -400 to $+150 \mathrm{~km} \mathrm{~s}^{-1}$, with respect to the companion galaxy (or from about -300 to $+300 \mathrm{~km} \mathrm{~s}^{-1}$, with respect to the QSO). The gas can be traced very far away from the QSO, even where no light is seen in the HST image, as already seen in Fig. 11. Even with discrepancies between [OII] and $\mathrm{H} \alpha$ velocity fields, the global trend is to show positive velocities on both sides of the galaxy, and negative elsewhere. The [OIII] velocity field is slightly different, globally positive on the whole upper part of the field and negative West of the quasar. This reinforces the hypothesis of a highly ionized gas cloud traced by [OIII], detached from the galaxy to which [OII] and $\mathrm{H} \alpha$ are more related.

The extraction of the stellar velocity field, from the CaII absorption lines, is restricted to the companion galaxy. Our measurements are presented in Fig. 12 (top left), ranging from -150 to $300 \mathrm{~km} \mathrm{~s}^{-1}$. The difference with the gas velocity field is striking, with almost reverse velocities, positive between the position of the galaxy and the quasar, and negative elsewhere. Taking into account the elongation of the VIMOS PSF, the rotation axis probably matches the major axis of the galaxy.

Clearly, gas and stellar dynamics in the companion galaxy are not related. Either the gas and the stars from which we measure the dynamics are located in physically different regions seen at the same position on the plane of the sky due to projection effects, or the gas and the stellar spectral features are formed in the same location. The first hypothesis would fit with the idea that HE 0450-2958 is embedded in a large cloud of gas ionized by the QSO radiation field at very large distances. The second hypothesis would rather indicate a violent shock between galaxies with the possible disruption of the host galaxy.

\section{Ionized gas and radio jets}

Ionized gas is present in a large volume around HE 0450-2958, as shown both by the slit and IFU observations. Two questions thus arise: (1) where does the gas come from? (2) How can it be so highly ionized as far away as $30 \mathrm{kpc}$ from the QSO?

Both questions may find an answer in the context of a collision between the companion galaxy and the putative quasar host, dispersing the gas and allowing the hard radiation field to reach large distances from the nucleus because of the very irregular gas (and possibly dust) distribution around the QSO.

The immediate surroundings of the QSO obviously contain some gas (such as the blob), but are also sufficiently transparent to let the QSO radiation field escape to large distances.

The detection of an extended gas distribution in the [OII] $3727 \AA$ line tends to weaken the hypothesis that the QSO host galaxy might remain undetected because of strong absorption by dust. Indeed, if the whole host was embedded in (or hidden behind) a huge dust cloud, it is difficult to understand how radiation at such short wavelengths might be detected throughout the system.

Deep infrared observations, aimed at testing this hypothesis are currently under analysis. However the presence of dust cannot be ruled out for the moment, and in this case the radiation of the quasar might evaporate the dust in some loci, producing patchy and highly ionized gas clouds.

Whatever the origin of the gas patches, a possible source of ionization in addition to the quasar radiation itself is a radio jet/cloud interaction, as already proposed by Feain et al. (2007). In Fig. 13 we reproduce their $6208 \mathrm{MHz}$ ATCA radio data, overlaid on the HST image and on the VIMOS [OIII] image. If dust was present on the path of the radio jets and lobes, appropriate correction for reddening might shift the ionization source for these regions from AGN to shock-induced ionization, these shocks then being caused by the jet. As seen from Fig. 13, there is a clear alignment between the radio lobes and the [OIII] emission. The alignment is found only between the radio data and the $[\mathrm{OIII}]$ data, rather than with $[\mathrm{OII}]$ and $\mathrm{H} \alpha$, suggesting that the radio jets are spatially related to high level ionization clouds, and not to star forming regions. However, as the flux used in the radio-to-infrared flux ratio estimated by Feain et al. (2007) concerns only the central peak of radio emission, this leaves their conclusions basically unchanged.

In addition, the strong spatial correlation between the radio jet and the $[\mathrm{OIII}]$ emission suggests that the jet is either the source of ionization or an efficient cleaner of the surroundings of the QSO, allowing its radiation to escape from the central region and to ionize much more distant gas clouds.

\section{Discussion}

Our new VLT 2D and 3D spectroscopic observations provide further evidence that the enigmatic QSO HE 0450-2958 lies in a strongly perturbed environment.

1. The putative host remains undetected, the companion galaxy is strongly distorted, with a gas velocity field apparently unrelated to the stellar one. 


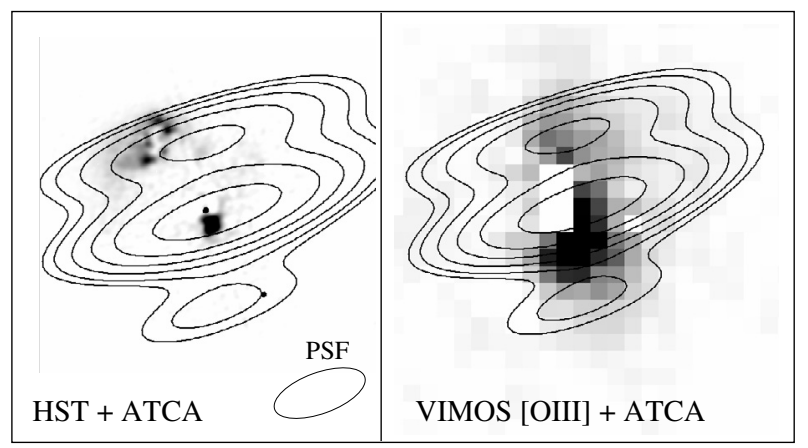

Fig. 13. Radio map obtained from the ATCA observations of Feain et al. (2007), overlaid on the deconvolved HST image (left) and on the VIMOS [OIII] image (right). Note the strong correlation between the radio structures and the [OIII] image and the misalignment with respect to the companion galaxy.

2. The whole system seems embedded in ionized gas, as best shown by the extended distribution of the [O II] emission.

3. Radio emission shows a peak at the position of the QSO as well as two roughly symmetrical lobes, one of them being located close to the companion galaxy.

4. Several clouds of highly ionized gas (those with the highest $[\mathrm{O} \mathrm{III}] /[\mathrm{O} \mathrm{II}]$ ratio) are found along the radio axis, extending from the immediate vicinity of the QSO (i.e. the "blob") up to more than $30 \mathrm{kpc}$.

5. These gas clouds appear to be ionized either by the QSO radiation itself or by shocks associated with jets.

6. This long range ionization suggests that the surroundings of the QSO are basically transparent to UV radiation, at least in two directions (along the radio axis and towards us - we recall that the blob spectrum shows no reddening - M05).

7. The facts that the radio lobe and long range ionization are stronger in the $\mathrm{S}-\mathrm{W}$ direction (i.e. towards the companion galaxy) than in the opposite direction might be due to the fact that the gas cloud immediately N-E of the QSO (the blob) absorbs most of the energy emitted in that direction. It may also be explained by a larger amount of gas in the direction of the companion galaxy than on the other side of the QSO/blob.

8. Several of these observations may find an explanation in the context of a collision which would have dispersed matter in a large volume around the QSO and companion galaxy.

Since HE 0450-2958 is a strong infrared emitter (IRAS point source catalog, de Grijp et al. 1987; Low et al. 1988), dust should be present in its surroundings, but the spatial resolution of the IRAS observations used for this identification does not allow us to locate precisely its origin. Kim et al. (2007) explained why this strong infrared emission cannot be caused by star formation in the companion galaxy: If the IRAS measured infrared fluxes were to be associated with star formation, they would imply a star formation rate of almost a thousand $M_{\odot} / \mathrm{yr}$, while the strength of the [OII]3727 $\lambda$ line in the optical FORS spectra of the companion galaxy only gives a maximum of $\sim 10 M_{\odot} / \mathrm{yr}$. Accordingly, the strong infrared emission is thus most probably not associated with the companion galaxy, but rather with the quasar itself. A simple explanation would be a dust torus around the accretion disk. The fact that the blob is unreddened suggests that either this torus is quite compact (at most a few hundred parsecs in order not to cover the blob) or the blob lies in front of it. The dust torus should also be inclined at an intermediate angle with respect to the line-of-sight. Indeed, an edge-on torus can be ruled out as it would mask the QSO. Similarly, a nearly face-on torus would hardly be compatible with the fact that the QSO UV radiation reaches projected distances as large as $30 \mathrm{kpc}$.

The fact that the UV radiation field of the QSO, after having left its immediate surroundings, can escape freely to large distances suggests that, at the kpc scale, the QSO neighbourhood is basically transparent in several directions. Even if we have no evidence for the complete absence of a host galaxy, this transparency is much easier to explain if the QSO does not lie in a massive host. Indeed, if such a galaxy was present in an environment so strongly perturbed by gravitational interactions, one would expect starburst regions with large amounts of gas and dust, as can be seen in the companion galaxy. Such a medium would not likely be transparent enough to explain the long range ionization. The present observations might thus reinforce the hypothesis of an undermassive host, if any at all.

On the other hand, the analysis of radio by Feain et al. (2007) seems to point towards some star formation around the quasar, leaving the possibility of a dust enshrouded host galaxy, explaining its non-detection in the optical, a dust medium which could have been swept in several directions by the radio jets, allowing the observed long range ionization by the powerful quasar.

Acknowledgements. G.L. is a teaching assistant supported by the University of Liege. G.L. and P.M. acknowledge support from Prodex 90195 (ESA/PPS Science Policy, Belgium). F.C. is supported by the Swiss National Science Foundation (SNSF).

\section{References}

Baldwin, J., Phillips, M., \& Terlevich, R. 1981, PASP, 93, 5

Courbin, F., Magain, P., Kirkove, M., \& Sohy, S. 2000, ApJ, 539, 1136

Courbin, F., Letawe, G., Magain, P., Wisotzki, L., Jablonka, P., et al. 2002, A\&A, 394,863

de Grijp, M. H. K., Lub, J., \& Miley, G. K. 1987, A\&AS, 70, 95

Djorgovski, S. G., Courbin, F., Meylan, G., Sluse, D., Thompson, D., et al. 2007, ApJ, 662, L1

Eigenbrod, A., Courbin, F., Meylan, G., Vuissoz, C., \& Magain, P. 2006, A\&A, 451,759

Eigenbrod, A., Courbin, F., \& Meylan, G. 2007, A\&A, 465, 51

Feain, I., Papadopoulos, P., Ekers, R., \& Middelberg, E. 2007, ApJ, 662, 872

Haehnelt, M., Davies, M., \& Rees, M. 2006, MNRAS, 366, 22

Hoffman, L., \& Loeb, A. 2006, ApJ, 638, 75

Kim, M., Ho, L., Peng, C., \& Im, M. 2007, ApJ, 658, 107

Letawe, G., Courbin, F., Magain, P., et al. 2004, A\&A, 424, 455

Letawe, G., Magain, P., Courbin, F., et al. 2007, MNRAS, 378, 83

Low, F. J., Cutri, R. M., Huchra, J. P., \& Kleinman, S. G. 1988, ApJ, 327, L41

Marconi, \& Hunt 2003, ApJ, 589, 21

Magain, P., Courbin, F., \& Sohy, S. 1998, ApJ, 494, 452

Magain, P., Letawe, G., Courbin, F., et al. 2005, Nature, 437, 381 (M05)

Merritt, D., Storchi-Bergmann, T., Robinson, A., et al. 2006, MNRAS, 367, 1746

Veilleux, S., \& Osterbrock, D. 1987, ApJS, 63, 295

Zhou, X., Yang, F., Lü, X., \& Wang, J.-M. 2007, AJ, 133, 432 